\title{
SMART Platforms: Building The App Store for Biosurveillance
}

\author{
Kenneth D. Mandl*

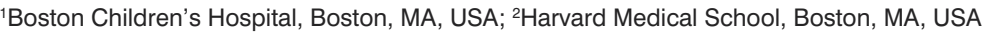

\section{Objective}

To enable public health departments to develop "apps" to run on electronic health records (EHRs) for (1) biosurveillance and case reporting and (2) delivering alerts to the point of care. We describe a novel health information technology platform with substitutable apps constructed around core services enabling EHRs to function as iPhone-like platforms.

\section{Introduction}

Health care information is a fundamental source of data for biosurveillance, yet configuring EHRs to report relevant data to health departments is technically challenging, labor intensive, and often requires custom solutions for each installation. Public health agencies wishing to deliver alerts to clinicians also must engage in an endless array of one-off systems integrations.

Despite a $\$ 48 \mathrm{~B}$ investment in HIT, and meaningful use criteria requiring reporting to biosurveillance systems, most vendor electronic health records are architected monolithically, making modification difficult for hospitals and physician practices. An alternative approach is to reimagine EHRs as iPhone-like platforms supporting substitutable apps-based functionality. Substitutability is the capability inherent in a system of replacing one application with another of similar functionality.

\section{Methods}

Substitutability requires that the purchaser of an app can replace one application with another without being technically expert, without requiring re-engineering other applications that they are using, and without having to consult or require assistance of any of the vendors of previously installed or currently installed applications. Apps necessarily compete with each other promoting progress and adaptability.

The Substitutable Medical Applications, Reusable Technologies (SMART) Platforms project is funded by a $\$ 15 \mathrm{M}$ grant from Office of the National Coordinator of Health Information Technology's Strategic Health IT Advanced Research Projects (SHARP) Program. All SMART standards are open and the core software is open source.

The SMART project promotes substitutability through an application programming interface (API) that can be adopted as part of a "container" built around by a wide variety of HIT, providing readonly access to the underlying data model and a software development toolkit to readily create apps. SMART containers are HIT systems, that have implemented the SMART API or a portion of it. Containers marshal data sources and present them consistently across the SMART API. SMART applications consume the API and are substitutable.

\section{Results}

SMART provides a common platform supporting an "app store for biosurveillance" as an approach to enabling one stop shopping for public health departments - to create an app once, and distribute it everywhere.

Further, such apps can be readily updated or created - for example, in the case of an emerging infection, an app may be designed to collect additional data at emergency department triage. Or a public health department may widely distribute an app, interoperable with any SMART-enabled EMR, that delivers contextualized alerts when patient electronic records are opened, or through background processes.

SMART has sparked an ecosystem of apps developers and attracted existing health information technology platforms to adopt the SMART API - including, traditional, open source, and next generation EHRs, patient-facing platforms and health information exchanges. SMART-enabled platforms to date include the Cerner EMR, the WorldVista EHR, the OpenMRS EHR, the i2b2 analytic platform, and the Indivo X personal health record. The SMART team is working with the Mirth Corporation, to SMART-enable the HealthBridge and Redwood MedNet Health Information Exchanges. We have demonstrated that a single SMART app can run, unmodified, in all of these environments, as long as the underlying platform collects the required data types. Major EHR vendors are currently adapting the SMART API for their products.

\section{Conclusions}

The SMART system enables nimble customization of any electronic health record system to create either a reporting function (outgoing communication) or an alerting function (incoming communication) establishing a technology for a robust linkage between public health and clinical environments.

\section{Keywords}

Electronic health records; Biosurveillance; Informatics; Application Programming Interfaces

\section{Acknowledgments}

This work was funded by the Strategic Health IT Advanced Research Projects Award 90TR000101 from the Office of the National Coordinator of Health Information Technology.

*Kenneth D. Mandl

E-mail: kenneth_mandl@harvard.edu 